\title{
CAPÍTULO 28: POTENCIAL FARMACOLÓGICO DA GOMA DO CAJUEIRO (Anacardium occidentale L.): UM BIOPOLÍMERO DO NORDESTE BRASILEIRO
}

\author{
CHAPTER 28: PHARMACOLOGICAL POTENTIAL OF CASHEW GUM \\ (Anacardium occidentale L.): A BIOPOLYMER FROM THE NORTHEAST BRAZIL
}

\author{
Márcia Nieves Carneiro da Cunha ${ }^{1}$; Maria Clara do Nascimento ${ }^{2}$, Carla Lêdo Morais ${ }^{3}$, Juanize Matias \\ da Silva Batista ${ }^{4}$, Vagne de Melo Oliveira ${ }^{5}$, Ana Lúcia Figueiredo Porto ${ }^{6}$
}

\begin{abstract}
Resumo
A goma de cajueiro (Anacardium occidentale L.) (GC) é composta principalmente por uma estrutura heteropolissacarídica altamente ramificada, constituída por galactose em sua cadeia principal com ligações $(1 \rightarrow 3)$ e cadeias laterais com ligações $(1 \rightarrow 6)$. A GC vem sendo utilizada em importantes processos biotecnológicos. Desta forma, a presente revisão objetiva realizar uma breve prospecção sobre a GC, suas propriedades biológicas e possíveis aplicações biotecnológicas. Para tal, foi realizado um levantamento dos artigos publicados sobre o tema disponíveis nas bases de dados Science direct, Scopus e Web of Science. Estudos demonstram o grande potencial de GC para aplicações farmacológicas e aplicação em processor de liberação controlada de drogas.
\end{abstract}

Palavras-Chave: Polissacarídeos, exsudatos vegetais, Gomas vegetais

\begin{abstract}
Cashew gum (Anacardium occidentale L.) (CG) is mainly composed of a highly branched heteropolysaccharide structure, consisting of galactose in its main chain with bonds $(1 \rightarrow 3)$ and side chains with bonds $(1 \rightarrow 6)$. A CG has been used in important biotechnological processes. Thus, this review aims to provide a brief prospection about $\mathrm{CG}$, its biological properties, and possible biotechnological applications. To this end, a survey of articles published on ready to use the topic in the databases Science direct, Scopus and Web of Science was carried out. Recent studies available in the literature suggest an enormous potential and wide applicability of this polymer and pharmacological and application in a controlled drug release process.
\end{abstract}

Keywords: Polysaccharides, vegetable exudates, vegetable gums

\footnotetext{
${ }^{1}$ Doutora em Biotecnologia, Universidade Federal Rural de Pernambuco, marcianieves@yahoo.com.br

${ }^{2}$ Bacharelado em Ciências Biológicas, Universidade Federal Rural de

Pernambuco, mclaranaascimento@ @otmail.com

${ }^{3}$ Pós-Graduação em Biociência Animal, Universidade Federal Rural de Pernambuco, carlaledo@ yahoo.com.br

${ }^{4}$ Doutora em Biociência Animal, Universidade Federal Rural de Pernambuco, juanizematias@yahoo.com.br

${ }^{5}$ Doutor em Ciências Biológicas, Universidade Federal Rural de Pernambuco, vagne_melo@ @hotmail.com ${ }^{6}$ Doutora em Engenharia Química, Professora Titular da Universidade Federal Rural de Pernambuco, analuporto@yahoo.com.br
} 
CUNHA, M. N. C. et al.

\section{Introdução}

O cajueiro (Anacardium occidentale L.) é uma planta nativa do nordeste brasileiro com considerável capacidade adaptativa a solos de baixa fertilidade, a temperaturas elevadas e ao estresse hídrico (SERRANO e PESSOA, 2016). A produção do caju no Brasil está concentrada na região Nordeste, e possui elevada importância socioeconômica para a Região, principalmente para o semiárido, por gerar postos de trabalho e renda na época mais seca do ano (BRAINER e VIDAL, 2018) e por ser um fruto fornecedor de matéria-prima para a fabricação de vários subprodutos (ALENCAR et al., 2018).

No Nordeste brasileiro, dada a importância econômica desta cultura, no ano de 2019 a área cultivada da planta foi de 434.614 hectare com uma produtividade para castanha de caju de 319 $\mathrm{kg} /$ hectare (CONAB, 2020). A goma do cajueiro (GC) é um coproduto do agronegócio do caju com grande potencial para aplicação em diferentes áreas industriais (PORTO et al., 2017). Nesse contexto, indubitavelmente, o desenvolvimento de novos bioprodutos a base de polissacarídeos do cajueiro é uma alternativa para agregar valor à cajucultura, além de, possibilitar a obtenção de um produto à base de matéria prima regional com baixo custo/benefício (SILVA et al., 2013).

Polissacarídeos obtidos a partir de várias fontes naturais têm sido amplamente utilizados na indústria, principalmente nas áreas alimentícia, farmacêutica e cosmética, além de movimentarem inúmeros trabalhos de pesquisa (Lima, Maia e Lima, 2013). Estudos sobre a GC despertam cada vez mais interesse da comunidade cientifica, visto que, este polissacarídeo possui características reológicas que favorecem sua aplicação biotecnológica. Desta forma, esta revisão teve por objetivo realizar uma breve prospecção sobre a GC, seu aspecto estrutural, obtenção e purificação, suas propriedades farmacológicas e aplicações biotecnológicas.

\section{Desenvolvimento}

\section{Cultura do Cajueiro (Anacardium occidentale L.) - Aspectos Econômicos}

O cajueiro (Anacardium occidentale L.) é uma planta nativa do Nordeste brasileiro, que apresenta uma grande capacidade adaptativa a solos de baixa fertilidade, a temperaturas elevadas e ao estresse hídrico (EMBRAPA, 2016). Características que favoreceram a difusão do cultivo desta planta em toda Região Nordeste, tornando-o uma importante fonte de renda para essa região. Para a comercialização o principal produto do cajueiro é a amêndoa da castanha-de-caju, localizada no interior da castanha. 
CUNHA, M. N. C. et al.

Segundo dados da CONAB (Companhia de Nacional de Abastecimento) dentre os Estados do Nordeste produtores de castanha-de-caju in natura, o Estado do Ceará destaca-se como o maior produtor, com uma produção de aproximadamente 87 mil toneladas em 2019, representando 62,4 \% do total de produção Nacional estimada em 139.3 mil toneladas. O Ceará é seguido pelos Estados do Piauí e do Rio Grande Norte com produções aproximadas de 21,6 e 16,8 mil toneladas em 2019, respectivamente.

Em um quadro de produção mundial da castanha, o Brasil está entre os dez maiores produtores, estando atrás de países como: Vietnã, Índia e Costa do Marfim (FAO, 2017). No ano de 2016 o Brasil situou-se como quinto maior exportador de castanha de caju sem casca, com uma participação de $3,1 \%$ do total global exportado naquele ano, o principal país importador deste produto são os Estados Unidos, seguido da Holanda e Alemanha (CONAB, 2019).

Como dito anteriormente a amêndoa da castanha-do-caju é principal produto comercial extraído do cajueiro, entretanto, praticamente todas as partes da planta podem ser aproveitadas, da castanha também pode ser extraída a película que reveste a amêndoa, rica em taninos utilizados na indústria química de tintas e vernizes. Já o pedúnculo do caju (pseudofruto) é processado por indústrias para a obtenção do suco ou da polpa congelada, a ser utilizada na fabricação de sucos, cajuínas e outras bebidas, na fabricação de doces e na alimentação animal, além disso, o caju inteiro (pedúnculo com castanha) é comercializado in natura em feiras e supermercados. Outras partes dessa planta também são utilizadas, as cascas das árvores e as folhas, são fontes de tanino e goma de interesse biotecnológico.

\section{A Goma do Cajueiro (GC)}

De acordo com Licá et al. (2018) as gomas são polímeros naturais, formados por unidades de monossacarídeos em arranjos lineares ou ramificados, pertencentes à classe dos carboidratos. São substâncias translúcidas, inodoras, insípidas, não tóxicas, hidrofílicas, amorfas, com propriedades coloidais, com funções espessantes (ligação com moléculas de água), gelificantes (construção de rede, envolvendo zonas de ligação), emulsificantes, estabilizantes e aglutinantes. Apresentam também, capacidade de controle de cristalização, inibição de sinerese, encapsulação e formação de filmes. As gomas exsudadas são produzidas pelas células epiteliais de plantas quando o córtex é agredido por injúrias físicas ou ataque microbiano. A produção do exsudado gomoso é um mecanismo de defesa destas plantas que crescem em áreas semiáridas (ANDRADE et al., 2013). Vários vegetais possuem a capacidade de produzir gomas, dentre os quais merece destaque o cajueiro (Anacardium occidentale L.). 
CUNHA, M. N. C. et al.

A goma de cajueiro (GC) é um heteropolissacarídeo aniônico complexo altamente ramificado obtido a partir do exsudado da casca ou do caule da árvore, é composto principalmente de $\beta$ - D- galactose (72\%), seguida de $\alpha$ - D- glicose

(14\%), arabinose $(4,6 \%)$, ramnose $(3,2 \%)$ e ácido glicurônico $(4,6 \%)$ e sua cadeia principal contém unidades de galactose unidas por elos do tipo $\beta(1 \rightarrow 3)$ e na cadeia lateral $\beta(1 \rightarrow 6)$ (QUELEMES et al., 2017), como pode ser observado na Figura 1. Segundo Paula et al. (2011) as concentrações dos constituintes da goma podem variar de acordo com a época do ano, além de fatores sazonais que a planta esteja sujeita no momento da extração do exsudato.

Este polissacarídeo possui algumas características atraentes para uso biotecnológico por ser não tóxico, hidrofílico, biocompatível e biodegradável, possuindo propriedades semelhantes à goma arábica em relação ao peso molecular, teor de ácido urônico e mesmo tipo de unidades monossacarídicas (PAULA et al., 2011).

Figura 1. Estrutura da goma de cajueiro (Anacardium occidentale, L.)

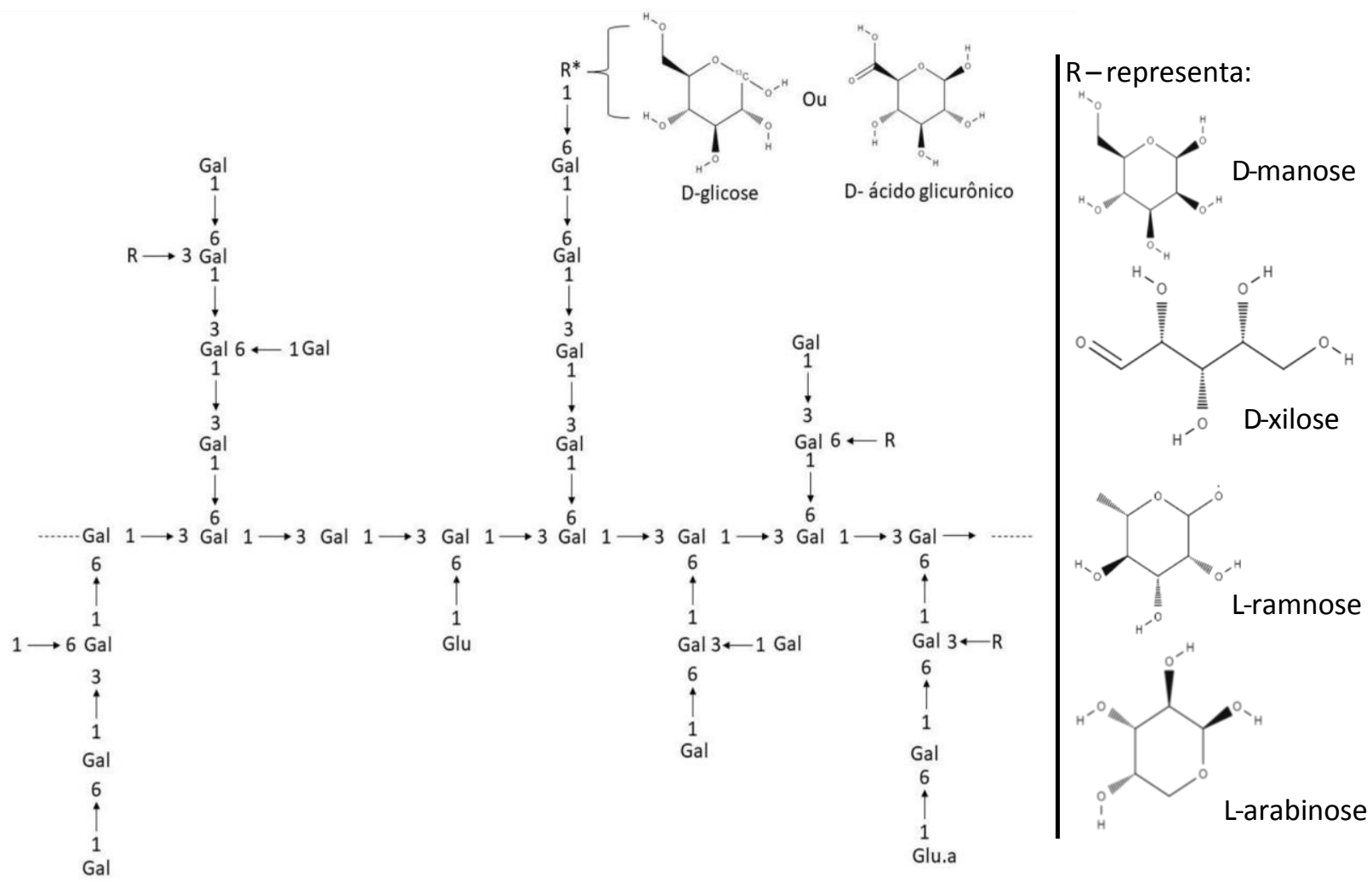

Fonte: Adaptado de Lima, Maia e Lima, 2013.

$\mathrm{Na}$ área de alimentos, a goma de cajueiro, especificamente, apresenta potencial de aplicação como aditivo alimentar na forma de espessante para sucos e refrescos, emulsificante para molhos e saladas e material de parede para microcápsulas (FURTADO et al., 2013). A GC também possui a capacidade de ser usada como aglutinante para comprimidos, hidrogéis 
CUNHA, M. N. C. et al.

superabsorventes, agentes gelificantes, como intensificadores de viscosidade, como surfactantes, revestimento agente para microencapsulação, e para liberação controlada de drogas, além disso, a GC apresenta diferentes propriedades farmacológicas, tais como: atividade antitumoral, antioxidante, anti-inflamatória e cicatrizante, antibacteriana e antifúngica. Como a goma do cajueiro possuí estrutura muito semelhante à da goma arábica. Pressupõe-se, portanto, que essa goma tenha potencialmente as mesmas aplicações da goma arábica (ARAÚJO et al., 2018). Além da semelhança estrutural (ramificações) e química (açúcares componentes) com a goma arábica, a GC tem um importante diferencial: sua alta disponibilidade na região Nordeste do território brasileiro, podendo gerar lucros no período da entressafra do caju (ANDRADE et al., 2013).

\section{Extração e purificação da Goma do Cajueiro}

A forma mais utilizada para extração da goma bruta exsudada do cajueiro é através do método físico onde é realizada uma incisão no tronco da planta e um exsudato que varia de amarelo pálido a marrom avermelhado é então coletado, e seco por exposição ao sol até perder a aderência e tornar-se duro e quebradiço, visando facilitar a manipulação e etapas posteriores de purificação. A outra forma de extração da goma é quimicamente pela ação de derivados do ácido benzoico (NAYAK et al., 2019).

Após a extração, a purificação da goma bruta é uma importante etapa realizada visando remover as impurezas da amostra, visto que, estas podem afetar as propriedades do polímero. As etapas de purificação da GC foram descritas inicialmente por Costa, Paula e Rodrigues em 1996, entretanto, o processo de purificação descrito por esses autores sofreu algumas modificações ao longo dos anos, como exemplo a secagem do material utilizando liofilização e o emprego de técnicas de cromatografia para confirmar a pureza da goma obtida após as etapas de purificação, contudo, as etapas mais importantes do processo continuam inalteradas (Figura 2). Para purificação, inicialmente a goma bruta é triturada e dissolvida em água destilada na proporção de $4 \mathrm{~g}$ de goma bruta para cada $100 \mathrm{~mL}$ de água destilada (GC extrato bruto) após completa dissolução essa solução apresentará pH ácido, o qual é corrigido pela adição de uma solução concentrada de cloreto de sódio para ocorrer a substituição de cátions por sódio. $\mathrm{O}$ ajuste de $\mathrm{pH}$ é um dos fatores mais críticos do processo. Valores de $\mathrm{pH}$ menores do que 6.0 diminuem a solubilidade da GC, pois nestas condições existe maior probabilidade de formação de pontes de hidrogênio entre as moléculas com a consequente agregação das partículas. Se o pH é elevado acima de 7,0, a solução e a goma obtida tornam-se escuras, provavelmente devido a solubilização de impurezas pouco solúveis em meio ácido. O GC extrato bruto é então filtrado, o sobrenadante é descartado, e o filtrado é precipitado com solventes voláteis, como acetona e 
etanol. O excesso de cloreto de sódio é removido através de lavagens sucessivas com água destilada, acetona e etanol e novamente sujeito a filtração e processos de precipitação. Para garantir a formação de sal de sódio da goma de caju, o pó obtido é ressuspendido em água destilada e passar por coluna cromatográfica, posteriormente o material é liofilizado, o pó seco é chamado de goma de caju purificada e será armazenada a baixas temperaturas. A figura 2 apresenta um esquema simplificado da purificação da GC.

Figura 2. Esquema simplificado com principais etapas de purificação da goma do cajueiro (Anacardium occidentale L.)

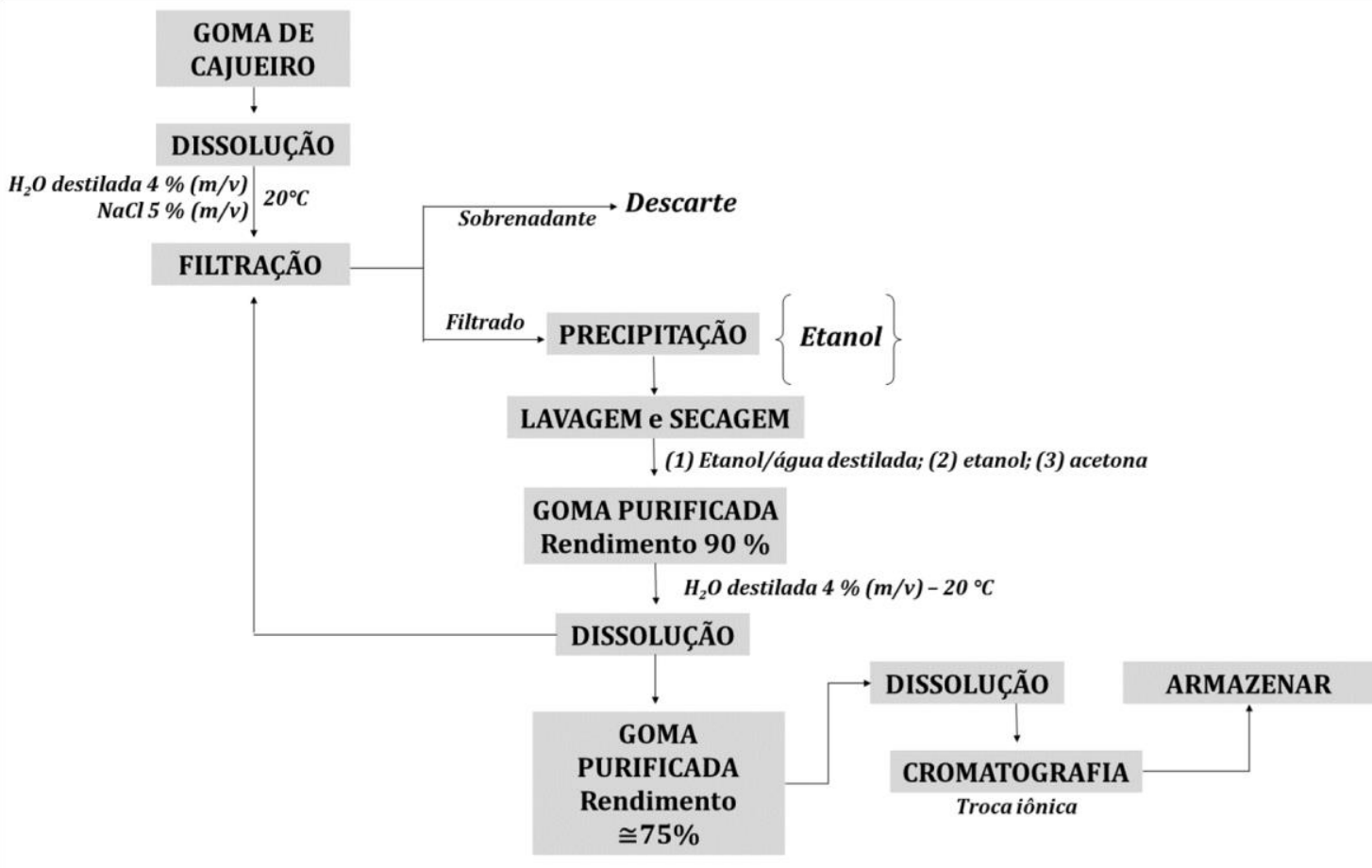

Fonte: Adaptado de Ribeiro et al., 2016

\section{Principais propriedades farmacológicas da Goma do Cajueiro}

A utilização da goma do cajueiro como medicamento natural é realizada a décadas com base no conhecimento popular em várias regiões do Nordeste brasileiro, onde é utilizada comumente pela população, como anti-inflamatório, agente cicatrizante de feridas, antidiarreico, gastroprotetor, dentre outros usos. O uso popular da goma vem estimulando pesquisas científicas com objetivo de confirmar tais propriedades atribuídas ao exsudato de cajueiro.

\section{Formulações biotecnológicas para Sistema de liberação de fármacos utilizando goma de} cajueiro

Os sistemas de liberação controlada possuem dois objetivos principais: manter constante a concentração sanguínea de uma determinada droga, assegurando uma maior 
CUNHA, M. N. C. et al.

biodisponibilidade, e reduzir os efeitos colaterais, realçando, assim, a adesão do paciente ao tratamento com um menor número de dosagens requeridas (HENRIQUE, FALCARE E

LOPES, 2006). Já foram descritos na literatura diferentes sistemas, tais como: lipossomas, as bombas osmóticas, os revestimentos entéricos, os sistemas transdérmicos, os prófármacos, e os sistemas matriciais poliméricos, nos quais proporcionam uma liberação com um tempo maior do fármaco no organismo especialmente no tecido ou órgão-alvo quando comparado com o convencional, tornando-se alvo de grande interesse das indústrias farmacêuticas (DE OLIVEIRA ALENCAR et al., 2018).

De acordo com Bizerra e Silva (2016) os sistemas poliméricos para liberação controlada representam alternativa para incorporação de substâncias ativas e apresentam vantagens como: (1) maior controle da liberação do princípio ativo; (2) redução de doses tóxicas e subterapêuticas; (3) monitoramento dos níveis de fármaco no sitio de aplicação; (4) obtenção de altas concentrações de fármaco no local a ser tratado, quando comparadas com os níveis plasmáticos após administração oral; e (5) direcionamento do princípio ativo a alvos específicos. Diminuindo o intervalo de dosagem e reduzindo efeitos colaterais indesejados uma vez que utiliza uma menor quantidade do princípio ativo, resultando em menor custo.

Substâncias naturais, como gomas de polissacarídeos, dentre essas a goma do cajueiro (GC), oferecem uma vantagem única no desenvolvimento e formulação de sistemas de entrega de medicamentos e são preferidos sobre os sintéticos (OLOGUNAGBA et al., 2017; NAYAK et al., 2019). Pesquisas recentes utilizando GC em sistemas de liberação de fármacos estão disponíveis na literatura, testando este biopolímero como excipiente na formulação de comprimidos, hidrogéis, biofilmes e ainda na produção de partículas poliméricas para a encapsulação de drogas.

\section{Discussão}

Para realizar uma breve prospecção a respeito das propriedades farmacológicas da goma do cajueiro (GC) e sobre sua aplicação no desenvolvimento de processos de entrega controlada de fármacos, foi realizada uma pesquisa em diferentes bases de periódicos (Tabela 1). $\mathrm{O}$ levantamento da literatura disponível sobre o tema foi realizado em julho de 2020, só foram considerados os artigos de pesquisa que utilizaram a unicamente a GC purificada ou a GC purificada em combinação com outros polissacarídeos naturais ou sintéticos, artigos científicos que avaliaram a atividade de outras partes da planta Anacardium occidentale L. (folha, casca, fruto) foram descartados, nenhuma restrição foi utilizada referente ao ano das publicações, 
CUNHA, M. N. C. et al.

apenas artigos de pesquisa foram selecionados, revisões de literatura ou outros documentos não foram considerados para a pesquisa.

Tabela 1. Base de Dados consultadas e termos de busca utilizados na revisão.

\begin{tabular}{lc}
\hline Base de dados & Endereço eletrônico \\
\hline Science Direct & https://www.sciencedirect.com/ \\
Scopus https://www.scopus.com/ \\
Web of Science & https://www.webofknowledge.com/ \\
Google Scholar & https://scholar.google.com/ \\
\end{tabular}

\section{Termos de busca}

("Cashew gum" OR “Anacardium occidentale") AND antitumor

("Cashew gum" OR “Anacardium occidentale") AND antiproliferative

("Cashew gum” OR “Anacardium occidentale") AND (Antimicrobial OR Antibacterial OR

Antifungal)

("Cashew gum" OR “Anacardium occidentale") AND Gastroprotective

("Cashew gum" OR “Anacardium occidentale") AND antidiarrheal

(“Cashew gum” OR “Anacardium occidentale”) AND “Drug delivery”

Fonte: Autor (2020)

\section{Principais propriedades farmacológicas da Goma do Cajueiro}

\section{Atividade Antitumoral}

A combinação da goma de cajueiro com outros oligossacarídeos e proteínas, exibiu atividade inibitória média de $88 \%$ p $<0,005$ contra um tumor sólido de sarcoma 180 implantado em camundongos (Mothé et al., 2008). O potencial antitumoral da goma de cajueiro (GC) in vitro e in vivo foi determinado por Barros et al. (2020), em seus estudos os citados autores avaliaram o nível de citotoxicidade da GC e o efeito antitumoral através do modelo de melanoma B16-F10. A inibição do tumor foi calculada com base no peso do tumor. Como resultado, o GC não demonstrou citotoxicidade in vitro, porém mostrou uma inibição tumoral significativa in vivo, com cerca de 36,9 a $43 \%$ de redução na massa tumoral, sem toxicidade para os órgãos.

O impacto do CG na morfologia e viabilidade celular com linhas celulares tumorais e não tumorais foi recentemente avaliado Ribeiro et al. (2020) utilizando microscopia de força atômica (MFA) e brometo de 3-(4,5-dimetil-2-tiazol)-2,5-difenil-2-H-tetrazólio (MTT), 
respectivamente. A atividade antiproliferativa foi confirmada para as linhas de células cancerígenas HCT116 (carcinoma colorretal), B16F10 (melanoma) e HL60 (leucemia promielocítica). Uma mudança na morfologia celular foi observada como uma rugosidade superficial aumentada para HL60. Nos resultados obtidos pelos autores não foi possível observar nenhum grau de citotoxicidade para linhas não-tumorais pela GC, a GC demostrou seletividade para células tumorais, sendo assim, pode-se considerar que a GC apresenta grande potencial antitumoral e é um biomaterial promissor para estudos futuros, desta forma, torna-se evidente a necessidade do estímulo por novas pesquisas neste campo de aplicação.

\section{Atividade Antimicrobiana}

Alguns autores relataram o potencial antibacteriano e antifúngico da goma de caju frente a diferentes espécies de micro-organismos. Torquato et al. (2004) testaram a atividade da goma de caju bruta e purificada frente a bactérias, leveduras e fungos. Esses autores observaram um aumento da atividade antimicrobiana na goma purificada em comparação com a goma bruta.

Estudos semelhantes foram realizados por Campos et al. (2012) visando analisar o mecanismo de ação antimicrobiana da goma de caju (bruta e purificada) por imagem de microscopia de força atômica (AFM), avaliaram o crescimento de oito diferentes espécies de bactérias na presença da goma. Os resultados indicaram fortes propriedades antimicrobianas da goma de caju puro contra todos os microrganismos testados, exceto Candida albicans e Lactobacillus acidophilus. Por outro lado, a goma de caju bruta apresentou apenas atividade antimicrobiana contra bactérias Gram-positivas (MRSA, MSSA, Listeria innocua $e$ Enterococcus faecium). As imagens de microscopia de Força atômica mostraram que a goma de caju purificada conduz ao colapso celular bacteriano indicando que a purificação da goma de caju afeta seu espectro antimicrobiano.

Mudanças na estruturais através da introdução de novos grupos funcionais, para adicionar caráter catiônico a GC, foram realizadas por Quelemes et al. (2017), que observaram forte atividade antimicrobiana contra cepas de Staphylococcus aureus resistente à meticilina. Os resultados descritos indicam que a goma de cajueiro purificada e estruturalmente modificada apresenta forte atividade antimicrobiana, entretanto, os relatos encontrados na literatura da ação isolada da goma (sem interação com outras substâncias) são antigos, e sugerem a necessidade de novos estudos. 
CUNHA, M. N. C. et al.

\section{Propriedades gastroprotetoras e antidiarreica da goma de cajueiro}

A goma de cajueiro (GC) é utilizada tradicionalmente no Nordeste brasileiro no tratamento de várias enfermidades, a exemplo de distúrbios do trato gastrointestinal, levando ao interesse da comunidade cientifica em investigar tais propriedades. Araújo et al. (2015) quando avaliaram a atividade da GC da contra diarreia aguda através do modelo de diarreia induzida por óleo de mamona e $\mathrm{PGE}_{2}$ em roedores, e o seu efeito contra diarreia secretora usando um modelo de secreção de fluidos em alças fechadas intestinais tratadas com toxina do cólera em camundongos vivos. Observaram uma excelente atividade antidiarreica da GC, visto que, nas concentrações avaliadas da goma a gravidade da diarreia aguda em ratos reduziu significativamente, além disto, a GC diminuiu o volume do óleo de mamona e $\mathrm{PGE}_{2}$ e a secreção de fluido intestinal induzida. Além disso, na diarreia secretora, o GC inibiu significativamente a secreção de fluidos intestinais. Os autores puderam concluir em seu estudo que a GC possui atividade antidiarreica nos modelos de diarreia aguda, inflamatória e secretora, o que poderia justificar seu uso tradicional no tratamento da diarreia no Nordeste do Brasil.

Um estudo realizado por Carvalho et al. (2015) teve como objetivo avaliar as propriedades gastroprotetoras da GC a partir de lesões estomacais induzidas por naproxeno (NAP) em Ratos Wistar machos e tratados com diferentes concentrações de GC (1, 3, 10 e $30 \mathrm{mg} / \mathrm{kg})$. O intestino delgado medial foi utilizado para avaliação das lesões macroscópicas e amostras do estômago e do intestino foram utilizadas para avaliação histológica em ensaios de glutationa (GSH), malonyldialde-hyde (MDA) e mieloperoxidase (MPO). Ratos adicionais foram utilizados para medir a secreção e muco gástrico. O pré-tratamento com a GC reduziu o dano macroscópico e microscópico induzido pelo NAP. A GC atenuou significativamente as alterações induzidas pela NAP nos níveis de MPO, GSH e MDA. Além disso, os autores observaram que os níveis de muco aderente retornaram aos valores normais após o tratamento com CG.

Resultados que corroboram com os apresentados por Nicolau et al., (2019) quando avaliaram a atividade da CG na mucosa esofágica humana (células provenientes de biópsias esofágicas humanas com refluxo não-erosivo) e de camundongos com Doença do refluxo gastroesofágico não-erosiva (DRGE) experimentalmente induzido. A GC aderiu à mucosa esofágica humana por até $1 \mathrm{~h}$. Nos estudos com animais, a GC melhorou os parâmetros da função barreira (TER e permeabilidade da mucosa) na mucosa do esôfago distal, e reduziu características inflamatórias do dano esofágico, conferindo proteção tópica da mucosa esofágica. Esses resultados confirmam o efeito protetor da GC contra os danos gastrointestinais por meio de mecanismos que envolvem a inibição da inflamação e o aumento da quantidade de muco aderente na mucosa. 
CUNHA, M. N. C. et al.

Tabela 2. Principais aplicações farmacológicas da goma do cajueiro (Anacardium occidentale L.) (GC).

\begin{tabular}{|c|c|c|c|}
\hline $\begin{array}{l}\text { Aplicações da } \\
\text { goma do } \\
\text { cajueiro }\end{array}$ & Métodos & Resultados & Autor e Ano \\
\hline \multirow{3}{*}{$\begin{array}{l}\text { Potencial } \\
\text { antitumoral }\end{array}$} & $\begin{array}{l}\text { Injetados } 200 \mathrm{mg} / \mathrm{kg} \\
\text { por } 7 \text { dias }\end{array}$ & $\begin{array}{l}\text { Atividade inibitória média de } 88 \% \\
\text { contra um tumor sólido de sarcoma } \\
180 \text { implantado em camundongos }\end{array}$ & Mothé et al., 2008 \\
\hline & $\begin{array}{l}\text { Modelo de melanoma } \\
\text { B16-F10 }\end{array}$ & $\begin{array}{l}\text { Inibição tumoral significativa in } \\
\text { vivo, com cerca de } 36,9 \text { a } 43 \% \text { de } \\
\text { redução na massa tumoral, sem } \\
\text { toxicidade para os órgãos }\end{array}$ & Barros et al., 2020 \\
\hline & MFA e MTT & $\begin{array}{l}\text { Atividade antiproliferativa } \\
\text { confirmada para as linhas de células } \\
\text { cancerígenas HCT116 (carcinoma } \\
\text { colorretal), B16F10 (melanoma) e } \\
\text { HL60 (leucemia promielocítica). }\end{array}$ & Ribeiro et al. 2020 \\
\hline \multirow[t]{4}{*}{$\begin{array}{l}\text { Potencial } \\
\text { Antibacteriana } \\
\text { e Antifúngica }\end{array}$} & $\begin{array}{l}\text { Atividade } \\
\text { antimicrobiana } \\
(\mathrm{NCCLS})\end{array}$ & $\begin{array}{l}\text { Aumento da atividade na goma } \\
\text { purificada em comparação com a } \\
\text { goma bruta }\end{array}$ & Torquato et al. 2004 \\
\hline & $\begin{array}{l}\text { Atividade antibacteriana } \\
\text { Microscopia de força } \\
\text { atômica (AFM) }\end{array}$ & $\begin{array}{l}\text { A goma de caju pura apresentou } \\
\text { fortes atividade antimicrobianas } \\
\text { contra os microrganismos testados }\end{array}$ & Campos et al., 2012 \\
\hline & $\begin{array}{l}\text { Modificação estrutural } \\
\text { (quaternização) da } \\
\text { goma e } \\
\text { teste frente a } \\
\text { Staphylococcus aureus } \\
\text { resistente à meticilina }\end{array}$ & Forte atividade antimicrobiana & Quelemes et al., 2017 \\
\hline & $\begin{array}{l}\text { Diarreia induzida por } \\
\text { óleo de mamona e } \mathrm{PGE}_{2} \\
\text { em roedores }\end{array}$ & $\begin{array}{l}\text { A gravidade da diarreia em ratos } \\
\text { reduziu significativamente e } \\
\text { diminuição da secreção de fluidos } \\
\text { intestinais }\end{array}$ & Araújo et al., 2015 \\
\hline \multirow[t]{2}{*}{$\begin{array}{l}\text { Atividade } \\
\text { gastroproteto } \\
\text { ras e } \\
\text { antidiarreica }\end{array}$} & $\begin{array}{l}\text { Tratamento de lesões } \\
\text { estomacais induzidas } \\
\text { por naproxeno (NAP) } \\
\text { em Ratos Wistar } \\
\text { machos }\end{array}$ & $\begin{array}{l}\text { O pré-tratamento com a GC reduziu } \\
\text { o dano macroscópico e } \\
\text { microscópico induzido pelo NAP }\end{array}$ & Carvalho et al., 2015 \\
\hline & $\begin{array}{l}\text { Avaliaram a atividade } \\
\text { da CG na mucosa } \\
\text { esofágica }\end{array}$ & $\begin{array}{l}\text { A GC reduziu características } \\
\text { inflamatórias do dano esofágico, } \\
\text { conferindo proteção tópica da } \\
\text { mucosa esofágica }\end{array}$ & Nicolau et al., 2019 \\
\hline
\end{tabular}




\section{Principais formulações biotecnológicas para Sistema de liberação de fármacos utilizando goma de cajueiro}

O sistema de liberação controlada de medicamentos é uma abordagem farmacológica moderna que tem como objetivo melhorar a eficácia do medicamento, aumentando a sua biodisponibilidade e diminuindo a toxicidade e possíveis efeitos colaterais para pacientes em tratamento (CRUZ et al., 2019; RICHTER et al., 2020). A micro e nanoencapsulação de moléculas bioativas em matrizes poliméricas naturais também tem despertado crescente interesse nas últimas décadas.

O estudo de novos polímeros naturais como agentes encapsulantes para microencapsulação é uma necessidade para atender exigências do mercado mundial e garante a disponibilidade de materiais alternativos àqueles já utilizados, preferencialmente de baixo custo, baixa toxicidade, ampla disponibilidade e biodegradabilidade. Estudos com a goma de cajueiro em associação com outros polímeros para obtenção de novos copolímeros têm sido amplamente realizados visando possíveis aplicações destes na indústria farmacêutica. Copolímeros de GC e L-lactídeo foram sintetizados para encapsular a anfotericina B (AMB), um antibiótico usado no tratamento de doenças fúngicas e leishmania com efeitos colaterais para pacientes em tratamento. Pode-se inferir que os copolímeros sintetizados apresentaram potencial como sistemas de nanocarreadores para AMB (RICHTER et al., 2020). Em outro estudo a GC enxertada com Nisopropilacrilamida (NIPA) foi utilizada para o encapsulamento da epirrubicina demonstrando bom potencial para uso de sistema de entrega deste medicamento (ABREU et al., 2016). Outro copolímero criado a partir da associação da GC com a goma de chicha (Sterculia striata) foi desenvolvido e testado como biomaterial para aplicação na indústria farmacêutica, apresentando características desejáveis e compatíveis com os materiais utilizados usualmente, ambos os polímeros naturais demonstraram ser excipientes promissores para uso como comprimidos e hidrogéis (DOS SANTOS FERREIRA et al., 2019). Da mesma forma, o potencial da associação da GC com a goma xantana como excipiente para comprimidos de ibuprofeno foi verificado através das formulações de comprimidos de matriz de ibuprofeno ( $200 \mathrm{mg}$ de ibuprofeno) contendo misturas variadas de GC e goma xantana preparadas por compressão direta. Todos os comprimidos testados demonstraram o mecanismo de transporte e a liberação de ibuprofeno envolveu difusão e erosão das matrizes de goma hidratada. Os estudos demonstraram o uso potencial da mistura desses polímeros como veículos para a entrega controlada de medicamentos (Fosu et al., 2016).

Hasnain et al., (2019) investigaram a utilidade da GC como excipiente farmacêutico em pastas dentárias contendo aceclofenaco para o tratamento da dor no tratamento da periodontite. 
CUNHA, M. N. C. et al.

Utilizando o CG com carbonato de cálcio (agente abrasivo), glicerina (umectante e co-solvente), parabeno metílico (conservante), lauril sulfato de sódio (surfactante) e cânfora (agente aromatizante) e $1 \% \mathrm{p} / \mathrm{p}$ de CA. As pastas dentárias formuladas demonstraram liberação sustentada de aceclofenaco por 6 horas, in vitro também revelando boa adesão à membrana mucosa oral. Essas pastas dentárias AC a $1 \%$ p / p podem ser usadas no tratamento eficaz da inflamação e dor dentárias através da administração local de AC durante um período prolongado no tratamento da periodontite.

Comprimidos de liberação controlada formulados com GC, GC reticulada e celulose microcristalina (MCC) foram preparados por compressão direta. Estudos in vivo para a formulação comercializada e otimizada mostraram que não houve diferença significativa entre os dois, confirmando o perfil de liberação sustentada. O estudo de estabilidade para formulação otimizada mostrou ainda melhores resultados. Pode-se concluir que gomas naturais e seus derivados podem ser efetivamente utilizados para a preparação de comprimidos de liberação sustentada (BHOSALE et al., 2015). Biofilmes de GC/PVA foram capazes de aprisionar inibidores de tripsina com atividade antimicrobiana obtidos de Platypodium elegans (PeIT) e Inga laurina (ILIT). No entanto, a atividade inibitória do PeIT imobilizado foi duas vezes maior que a observada para o ILIT. Além disso, os inibidores liberados mostraram alta estabilidade após 24 h de armazenamento, confirmando que os filmes GC/PVA são materiais versáteis e eficientes para serem utilizados como suporte à imobilização de biomoléculas (CRUZ et al., 2019).

Estudos estão sendo realizados com objetivo de identificar como ocorre a interação entre o polímero e a droga, como o estudo realizado por Cordeiro et al. (2017) para avaliar a interação entre o biopolímero, composto da associação GC com a quitosana, e o medicamento pilocarpina foi realizado por visando identificar como ocorre a liberação controlada da droga, de acordo com esses autores a GC interagiu com a pilocarpina, tendo um efeito térmico protetor sobre a droga.

Além do uso de polissacarídeos do cajueiro em associação com outros polímeros vegetais ou sintéticos, na literatura disponível nos bancos de dados utilizados nesta pesquisa foi possível identificar que mudanças estruturais na goma também são alvo de pesquisa, pois estas conferem diferença nas propriedades químicas e físicas da GC, como exemplo modificando sua solubilidade em água, dentre outras. Estudos realizados a partir da acetilação da goma, testou a capacidade desta na produção de nanopartículas e mostrou grande potencial como veículo em sistemas controlados de liberação de drogas, principalmente quando testado no nanoencapsulamento do alcalóide natural epiisopiloturina. As nanopartículas sintetizadas 
CUNHA, M. N. C. et al.

mostram grande potencial para o uso na liberação da epiisopiloturina. (DO AMARAL RODRIGUES et al., 2019). Da mesma forma, estudos foram realizados por Silva et al. (2019) também utilizando a GC acetilada como plataforma em sistemas de entrega de medicamentos tendo a insulina como droga modelo. No citado estudo as nanopartículas foram desenvolvidas através da técnica de complexação polieletrolítica e o resultado mostrou uma eficiência do encapsulamento da insulina de $52,5 \%$ e a estabilização eletrostática foi sugerida pelo potencial zeta de $+30,6 \mathrm{mV}$. A liberação sustentada de insulina foi observada por até $24 \mathrm{~h}$. Sugerindo que GC acetilado apresentou potencial como veículo para liberação sustentada de insulina por via oral. A indometacina, um conhecido um agente anti-inflamatório, também foi nanoencapsulada utilizando GC acetilada como material de parede e liberação controlada da droga foi observada por até 72 h (Pitombeira et al., 2015).

Biofilmes de GC/PVA foram capazes de aprisionar inibidores de tripsina com atividade antimicrobiana obtidos de Platypodium elegans (PeIT) e Inga laurina (ILIT). No entanto, a atividade inibitória do PeIT imobilizado foi duas vezes maior que a observada para o ILIT. Além disso, os inibidores liberados mostraram alta estabilidade após 24 h de armazenamento, confirmando que os filmes GC/PVA são materiais versáteis e eficientes para serem utilizados como suporte à imobilização de biomoléculas (CRUZ et al., 2019).

\section{Considerações Finais}

Muito se tem pesquisado em relação à produtos medicinais naturais e seu uso terapêutico. De acordo com os resultados obtidos na revisão de literatura a respeito das propriedades farmacológicas da goma do cajueiro (GC) e sua aplicação no desenvolvimento de novos bioprocesso para liberação controlada de fármacos foi possível inferir que os polissacarídeos exsudados do cajueiro possuem uma ampla atividade farmacológica, neste estudo foram descritos trabalhos que evidenciaram em seus resultados a potencialidade da CG para uso antitumoral e antiproliferativo, antimicrobiano, frente a fungos, bactérias e leveduras, gastroprotetor e antidiarreico.

No que se refere a aplicação da GC em processos biotecnológicos de liberação controlada de fármacos, estudos recentes relatam o uso da GC em combinação com outros polissacarídeos e CG estruturalmente modificada, como material para excipiente de fármacos, através de formulações de comprimidos, biofilmes, hidrogéis e micro e nanoencapsulamento de drogas, tais pesquisas apresentam resultados que demonstram o grande potencial deste biopolímero para aplicações biotecnológicas indicando o uso de gomas naturais como uma alternativa eficaz aos polímeros sintéticos. 
Entretanto, na presente pesquisa foi possível verificar um escasso número de publicações principalmente no que se refere ao potencial farmacológico da GC e de maneira geral, mostra a importância do incentivo ao do desenvolvimento de novas pesquisas sobre as propriedades e tecnológicas da GC, um polissacarídeo de baixo custo de obtenção e amplamente encontrado no Nordeste brasileiro.

\section{Referências}

ABREU, F. O., OLIVEIRA, E. F., PAULA, H. C., \& DE PAULA, R. C. Chitosan/cashew gum nanogels for essential oil encapsulation. Carbohydrate polymers, 89(4), 1277-1282, 2012.

ALENCAR, N. S., GONÇALVES, J. F., OLIVEIRA, E. A F., LUCENA, T. C., SOUSA, R. M. Produção da Castanha de Caju nas microrregiões do Ceará no período de 1993 a 2016. RECoDAF - Revista Eletrônica Competências Digitais para Agricultura Familiar v. 4, n. 1. ISSN: 2448-0452, 2018.

ANDERSON, D.M.W.; BELL, P. C. Structural analysis of the gum polysaccharide from anacardium occidentale. Analytica Chimica Acta, v79, p 185-197, 1975.

ANDRADE, K., DE CARVALHO, C. W., \& TAKEITI, C. Y. Goma de cajueiro (Anacardium occidentale): avaliação das modificações químicas e físicas por extrusão termoplástica. Polímeros, 23(5), 667-671, 2013.

ARAÚJO, T. S., COSTA, D. S., SOUSA, N. A., SOUZA, L. K., DE ARAÚJO, S., OLIVEIRA, A. P., ... \& MEDEIROS, J. V. R. Antidiarrheal activity of cashew GUM, a complex heteropolysaccharide extracted from exudate of Anacardium occidentale L. in rodents. Journal of ethnopharmacology, 174, 299-307, 2015

BARROS, A.B., MOURA, A.F., SILVA, D.A., OLIVEIRA, T.M., BARRETO, F.S., RIBEIRO, W.L.C., ALVES, A.P.N.N., ARAÚJO, A.J., MORAES FILHO, M.O., ILESG, B., MEDEIROS, J.V.R., MARINHO-FILHO, J.D.B. Evaluation of antitumor potential of cashew gum extracted from Anacardium occidentale Linn. International Journal of Biological Macromolecules Volume 154, 1 July 2020, Pages 319-328 
BHOSALE, R. R., OSMANI, R. A. M., \& MOIN, A. Natural gums and mucilages: a review on multifaceted excipients in pharmaceutical science and research. International Journal of Pharmacognosy and Phytochemical Research, 15(4), 2014.

BIZERRA, A., SILVA, V. Sistemas de liberação controlada: Mecanismos e aplicações. Revista Saúde e Meio Ambiente, v. 3, n. 2, p. 1-12, 2016.

BRAINER, M. S. DE C. P.; VIDAL, M. DE F. V. Cajucultura nordestina em recuperação. Caderno setorial ETENE, v. 54, n. Ano 3, 2018

CAMPOS, D. A., RIBEIRO, A. C., COSTA, E. M., FERNANDES, J. C., TAVARIA, F. K., ARARUNA, F. B., ... \& PINTADO, M. M. (2012). Study of antimicrobial activity and atomic force microscopy imaging of the action mechanism of cashew tree gum. Carbohydrate polymers, 90(1), 270-274

CARVAlHO, N. S., SILVA, M. M., SILVA, R. O., NICOLAU, L. A., SOUSA, F. B. M., DAMASCENO, S. R., ... \& MEDEIROS, J. V. R. (2015). Gastroprotective properties of cashew gum, a complex heteropolysaccharide of Anacardium occidentale, in naproxen- induced gastrointestinal damage in rats. Drug Development Research, 76(3), 143-151.

CARVAlHO, N. S., SILVA, M. M., SILVA, R. O., NICOLAU, L. A., SOUSA, F. B. M., DAMASCENO, S. R., ... \& MEDEIROS, J. V. R. (2015). Gastroprotective properties of cashew gum, a complex heteropolysaccharide of Anacardium occidentale, in naproxen- induced gastrointestinal damage in rats. Drug Development Research, 76(3), 143-151, 2015.

CONAB, Histórico Mensal Castanha-de-caju, Maio de 2020, acesso em 29 de julho de 2020 (https://www.conab.gov.br/info-agro/analises-do-mercado-agropecuario-eextrativista/analisesdo-mercado/historico-mensal-de-castanha-de-caju).

CORDEIRO, M. S. F., DA SILVA, C. M. B., VIEIRA, A. C. Q. D. M., NADVORNY, D., DE SÁ, L. L. F., DE SOUZA, F. R. L., ... \& RIBEIRO, A. J. Biopolymers and pilocarpine interaction study for use in drug delivery systems (DDS). Journal of Thermal Analysis and Calorimetry, 127(2), 1777-1785, 2017.

COSTA, S., RODRIGUES, J., \& DE PAULA, R. (1996). Monitorização do Processo de Purificação de Gomas Naturais: Goma do Cajueiro. Polímeros: Ciência e Tecnologia, 6, 4955.

CRUZ, M. V., JACOBOWSKI, A. C., MACEDO, M. L., BATISTA, K. A., \& FERNANDES, K. F. Immobilization of antimicrobial trypsin inhibitors onto cashew gum polysaccharide/PVA films. International journal of biological macromolecules, 127, 433-439, 2019.

DE OLIVEIRA ALENCAR, G., BEZERRA, C. A., SCHERF, J. R., DA SILVA, J. F., BARRETO, M. D. F. R., ALVES, M. P., \& DE CARVALHO MENDES, R. Liberação prolongada de fármacos e suas aplicações farmacológicas: revisão sistemática. Revista ECiência, v 6, 2018.

DO AMARAL RODRIGUES, J., DE ARAÚJO, A. R., PITOMBEIRA, N. A., PLÁCIDO, A., DE ALMEIDA, M. P., VERAS, L. M. C., ... \& FEITOSA, J. P. A. Acetylated cashew gumbased nanoparticles for the incorporation of alkaloid epiisopiloturine. International journal of biological macromolecules, 128, 965-972, 2019. 
DOS SANTOS FERREIRA, S. R., MESQUITA, M. V. N., DE SÁ, L. L. F., NOGUEIRA, N. C., DOS SANTOS RIZZO, M., SILVA-FILHO, E. C., ... \& RIBEIRO, A. B. Sustainable natural gums for industrial application: Physiochemical and texturometric evaluation. Journal of Drug Delivery Science and Technology, 54, 101306, 2019.

FOSU, M. A., OFORI-KWAKYE, K., KUNTWORBE, N., \& BONSU, M. A. Investigation of blends of cashew and xanthan gums as a potential carrier for colonic delivery of Ibuprofen. International Journal of PharmTech Research, 9(7), 369-380, 2016.

HASNAIN, M. S., RISHISHWAR, P., RISHISHWAR, S., ALI, S., \& NAYAK, A. K. Extraction and characterization of cashew tree (Anacardium occidentale) gum; use in aceclofenac dental pastes. International journal of biological macromolecules, 116, 1074$1081,2018$.

HENRIQUE, J. S.; FALCARE, R. S.; LOPES, P. S. Sistemas de liberação controlada. Pharmacia Brasileira, v. 56, p. 22, 2006.

LICÁ, I. C. L., DOS SANTOS SOARES, A. M., DE MESQUITA, L. S. S., \& MALIK, S. Biological properties and pharmacological potential of plant exudates. Food Research International, 105, 1039-1053, 2018.

LIMA, R. E. M., MAIA, L. K. R., \& LIMA, J. S. Produção de Goma a Partir do Cajueiro. Enciclopédia Biosfera, 9(17), 2093-2106, 2013.

MOTHÉ, C.G., DE SOUZA, I.A., CALAZANS, G.M.T. Antitumor activity of cashew gum from Anacardium Occidentale L., Agro Food Industry Hi-Tech 19 (6), pp. 50-52, 2008.

NAYAK, A. K., ANSARI, M. T., SAMI, F., BERA, H., \& HASNAIN, M. S. (2019). Cashew gum in drug delivery applications. In Natural Polysaccharides in Drug Delivery and Biomedical Applications (pp. 263-283). Academic Press.

NICOLAU, L. A., BATISTA-LIMA, F. J., SANTANA, A. P., SALES, T. M., CARMONETO, J. P., FREITAS, G. B., ... \& WOODLAND, P. J. Cashew gum, a biopolymer, topically protects oesophageal mucosa in non-erosive reflux disease: A promising translational study. Carbohydrate polymers, 226, 115205, 2019.

OLOGUNAGBA, M. O., JAIN, S., THANKI, K., SURESH, S., FURTADO, S., AZUBUIKE, C. P., \& SILVA, B. O. (2017). Extraction and Characterization of the Gum Exudate of Anacardium occidentale for its potential as an Excipient in Drug Delivery Systems. Trop J Nat Prod Res, 1(2):76-83, 2017.

PAULA, H. C.; SOMBRA, F. M.; DE FREITAS CAVALCANTE, R.; ABREU, F. O.;

DEPAULA, R. C. Preparation and characterization of chitosan/cashew gum beads loaded with Lippia sidoides essential oil. Materials Science and Engineering C., v. 31, n. 2, p. 173-178, 2011.

PITOMBEIRA, N. A., NETO, J. G. V., SILVA, D. A., FEITOSA, J. P., PAULA, H. C., \& DE PAULA, R. C. Self-assembled nanoparticles of acetylated cashew gum: Characterization and evaluation as potential drug carrier. Carbohydrate Polymers, 117, 610-615, 2015.

PORTO, B. C.; FURTADO, R. F.; CRISTIANINI, M. Caracterização de emulsão de goma de cajueiro modificada quimicamente para o microencapsulamento de D-limoneno. Embrapa Agroindústria Tropical-Boletim de Pesquisa e Desenvolvimento (INFOTECA-E), 2017. 
QUELEMES, P. V., DE ARAÚJO, A. R., PLÁCIDO, A., DELERUE-MATOS, C., MACIEL, J. S., BESSA, L. J., ... \& DA SILVA, D. A. Quaternized cashew gum: An anti-staphylococcal and biocompatible cationic polymer for biotechnological applications. Carbohydrate polymers, 157, 567-575, 2017.

RIBEIRO, F. D. O. S., DE FRANÇA DOURADO, F., SILVA, M. F. S., BRITO, L. M., PESSOA, C., DE LIMA, L. R. M., ... \& DA SILVA, D. A. (2020). Anti-proliferative profile of Anacardium occidentale polysaccharide and characterization by AFM. International Journal of Biological Macromolecules. Volume 156, 1 August 2020, Pages 981-987

RICHTER, A.R., CARNEIRO, M.J., DE SOUSA, N.A., (...), GOYCOOLEA, F.M., DE PAULA, R.C.M. Self-assembling cashew gum-graft-polylactide copolymer nanoparticles as a potential amphotericin B delivery matrix. International Journal of Biological Macromolecules, 152, pp. 492-502, 2020.

SERRANO, L. A. L.; PESSOA, P. F. A. P. Aspectos econômicos da cultura do cajueiro. Embrapa agroindústria tropical: Fortaleza-Ceará, 2016.

SILVA, E. D. L. V., DE JESUS OLIVEIRA, A. C., PATRIOTA, Y. B. G., RIBEIRO, A. J., VEIGA, F., HALLWASS, F., ... \& SOARES-SOBRINHO, J. L. Solvent-free synthesis of acetylated cashew gum for oral delivery system of insulin. Carbohydrate polymers, 207, 601608, 2019.

SILVA, R. A. O., MARQUES, L. G. A., DE FREITAS, R. M., DOS SANTOS, M. D. S. F., AVALCANTI DA SILVA FILHO, E., DO Ó PESSOA, C., \& SANTOS, M. R. D. M. C. Prospecção tecnológica: aplicação da goma do cajueiro (Anacardium occidentale) em nanotecnologia. Revista GEINTEC-Gestão, Inovação e Tecnologias, 3(4), 055-069, 2013.

TORQUATO, D. S., FERREIRA, M. L., SÁ, G. C., BRITO, E. S., PINTO, G. A. S., \& AZEVEDO, E. H. F. Evaluation of antimicrobial activity of cashew tree gum. World Journal of Microbiology and Biotechnology, 20(5), 505-507, 2004. 